\title{
EFFECTS OF A STAGGERED CONFIGURATION OF A TIDAL TURBINE ARRAY EMBEDDED INTO A BOUNDARY LAYER ON FLOW STRUCTURE
}

\author{
Ahmed Hassb Elkarem \\ Faculty of Engineering, \\ University of Khartoum, Khartoum, Sudan \\ Nina Nikora \\ Faculty of Engineering, \\ The University of Aberdeen, Aberdeen, United Kingdom
}

\begin{abstract}
There is a rapid development in tidal current turbines to provide a fair share of global energy requirements. Tidal turbines are being installed in arrays to maximise the energy extraction. The performance of the tidal turbines and their impact on the flow will depend on the layout. This study aims towards improving the understanding of the effect of a staggered layout i.e. lateral and longitudinal spacing between the turbines, on flow structure. Four sets of experiments have been conducted for a four model turbines array. Each of the lateral and longitudinal spacing in the array has been changed twice. Velocity measurements have been taken with an Acoustic Doppler Velocimeter (ADV). The measurements were taken in six cross-section.
\end{abstract}

Keywords: Tidal array, wakes, ADV, Turbulence intensit

\section{INTRODUCTION}

The oceans are direct and tremendous sources of sustainable energy. Oceans cover more than $70 \%$ of the earth surface, thus store huge amounts of energy in different forms. The main types of energy stored are kinetic energy (wave and currents), thermal energy, chemical energy (osmosis) and biomass energy [1-2].

Primarily, tidal power is viewed as two components. Potential power generated from the difference in vertical height between the high and low tides, and kinetic power caused by the movement of the tides.
Depending on the energy component, the tidal energy devices are classified into tidal currents (streams) devices, tidal barrages and dynamic tidal power [3-5]. Tidal energy barrages exploit the potential energy by constructing a dam to hold the water and thus employing the same working principle as hydroelectric generation [2,5]. Whereas the tidal current devices utilise the kinetic energy of the water currents in a similar principle to the wind turbines. The turbine blades are made of typically made of lightweight composites due to their high specific flexural stiffness [6-10]. Turbines can be classified depending on the blade axis of rotation relative to the current flow direction into, horizontal axis and vertical axis turbines $[2,11]$.

Tidal currents are concentrated within narrow channels with high kinetic energy enough to make the exploitation of the energy feasible [12]. Therefore, laying the stream turbines in arrays to make the most of the currents of vital importance [13].

Another motive to use multiple turbines is because the power capacity of an individual turbine is limited by the Lanchester-Betz theory. Accordingly, to maximise the power output at a certain cite, multiple turbines must be used, i.e. array. [13,14].

However, the concentration of the resource and the presence of multiple turbines in the array require the understanding of the space between the turbines in the array. The spacing between turbines affects the extent of the wakes expansion and recovery following each row of turbines [3,15]. Wakes are fundamental factor in understanding the extent of 
the energy exploitation within an array [15]. Since, they can affect the downstream device by reducing the velocity, increasing the turbulences, or creating asymmetrical flow profiles that results in an increased loading on some devices within the array. The investigation of the effect of the tidal turbines and tidal arrays on the wake has been an interest in many studies.

The wake structure of marine turbines has been studied both numerically and experimentally in the literature. Numerical methods are widely used because they provide inexpensive yet reasonable measurements for understanding the flow[16]. Sun et al. [17] used an absorption disk to simulate a tidal turbine using CFD effluent in one of the earliest researches into the effect of the energy extraction from tidal turbines on flow structure. More CFD studies on the effect of a turbine have been conducted, also simulating the turbine as a disk $[18,19]$. These studies examined the effect of the turbulence and the turbulent inflow on the wake recovery.

The higher the ambient turbulence levels, the more expeditiously the wake recover with downstream distance. Furthermore, the unsteady inflow has boosted the wake recovery as well. The same findings are also present in a recent study by Ahmadi.[20], where the effect of the upstream turbulence on the flow characteristics downstream of a laboratory-scale was investigated by using a hybrid Large Eddy Simulation/Actuator Line Modelling technique.

Harrison et al.[21] used a model to investigate the performance of individual turbines within a fiverow array of turbines and the wake recovery velocity within the array. They used a combined blade element theory (BEM), and Reynolds averaged Naiver-Stokes (RANS) to achieve this goal. Mulualem et al.[22] used a CFD based immersed body force method to investigate the effect of the wake interaction on the individual turbines within a staggered arrangement in a tidal current farm. They found that there should be a balance between the blockage effect and the wake interaction.

The blockage effect in the upstream resulting from the closely packed turbines could improve the performance of these turbines and the array, however, the wake interaction of those turbines might affect the downstream turbines thus affecting the power output of the array.

Staggered configurations might help in achieving the balance if there are proper bypass flow regions which benefit from the accelerated flow resulting from the blockage effect [22]. Bai et al.[23] used a numerical model that was verified with experimental data to investigate the performance of arrays in a staggered and aligned position.

They demonstrated that staggered arrays are better in term of power absorption than rectilinear arrays. Churchfield et al.[16] used a large eddy simulation of tidal turbines. They arrived at the same conclusion as the previous study that staggering the successive rows of turbines give higher power extraction.

Nonetheless, several experimental studies have been conducted on tidal turbines. These studies either used scaled model turbines or disks to model the turbines $[24,25]$ used scaled three-bladed model turbines to investigate the performance of tidal turbines by changing a few parameters such as the blockage ratio. Myers and Bahaj [26] used disk simulators to study the effect of the horizontal turbines on the wake structure, they concluded that the wake velocity is reduced in the near wake region.

However, in the far wake region, the wake velocity will depend on the mixing of turbulences. Moreover, they also studied how the proximity to the sea bed affects the wake recovery, and they found that the closer the disk to the sea bed, the more persisting the wake will be. Finally, they increased the turbulence by adding more roughness to the sea bed, but they realised that no reduction in the wake persistence could be achieved by increasing the seabed roughness. F. Maganga et al.[27] used model turbines to study the effect of horizontal tidal turbines on the flow and regarding the ambient intensity, they realised that increasing the turbulence intensity leads to a high rate of velocity recovery because of the formation of narrower wakes.

A more detailed study of the near wake region has been conducted in Tedds et al. work [28] They studied the near wake region of a tidal turbine by using ADV measurements and a tidal turbine model.

The authors focused on the effects of the turbine on the velocity field and the turbulent flow field. The study revealed the complexity of those fields, and how strong are anisotropic of the turbulence and they suggested avoiding near wake turbulence models based on the isotropy of the wakes.

Chen et al.[29] used an ADV to take measurements and completed a similar experiment to the previous study in [28]. However, the study was expanded to include the far wake region as well. The measurements were taken up to 20 diameters. 
From the experiment results, they showed that the velocity reduction is attributed to the blockage effect of the turbine rotor and stanchion.

Moreover, it was also shown that there is strong anisotropic wake turbulence which will ultimately influence the behaviour of other turbines if they are located in an array. Paul Mycek et al.[30,31] conducted a two-part study to examine the effect of ambient turbulence on tidal turbines.

The first part dealt with a single turbine and the second part with two turbines located axially one behind the other. They found that the higher turbulent intensity yields better performance in both cases, which is a similar finding to the previous study in [28], Stallard et al.[15] focused on investigating the effect of the lateral spacing between turbines in arrays on the wake developed.

They aimed to compare the wake characteristics of an isolated single rotor with different arrays of turbines. They found that for $3 \mathrm{D}$, the wake profile is similar to a single rotor whereas, for spacing less than $2 \mathrm{D}$, the wake starts to merge within $4 \mathrm{D}$ downstream.

Nuernberg \& Tao [3] used Particle Image Velocimetry (PIV) measurements to examine the wake characteristics of a staggered array layout of scaled tidal turbines.

They concluded that close spacing between turbines leads to a substantial reduction in the velocity recovery. Moreover, the wider the lateral spacing, the less significant the longitudinal spacing impact on the rate of velocity recovery.

Although the study of the effect of the tidal turbine array has been studied numerically but it experimentally has not been extensively studied. Some authors studied the impact of an array of three turbines such as [32,33].

However, to the author knowledge there has not been a study on the effect of the layout of a four turbines staggered array on the wake structure using an ADV device. This work will provide answers to the effect of layout of the wake through a set of experiments.

\section{EXPERIMENTAL TECHNIQUE}

All the tests were performed at the Fluids laboratory at the University of Aberdeen. The Flume is $0.3 \mathrm{~m}$ wide and $12.5 \mathrm{~m}$ long with a wall height of $0.46 \mathrm{~m}$. The first turbine is positioned approximately in the middle of the flume, at a distance $6 \mathrm{~m}$ away from the entrance of the flume to ensure that the flow is fully developed, as shown in Figure 1.

Nortek Acoustic Doppler Velocimeter (ADV), was used to obtain the velocity components and the turbulences statics. The device has a $10 \mathrm{MHz}$ receiving element and a sampling volume of $9 \mathrm{~mm}$. The velocity data were sampled at a rate of $25 \mathrm{HZ}$ and a sampling period of $90 \mathrm{~s}$, which resulted in 2250 samples at each point. The sampling volume and the sampling rate were based on the recommendation of the adv manual to get good results quality [34]. Moreover, the data collected had a signal to noise ratio (SNR) higher than a $15 \mathrm{db}$ and correlation coefficient higher than 75 .

\subsection{Turbine arrangement}

Four model turbines were used in this experiment as shown in Figure 1 Top view of the flume and the position of the first turbine in the array

. Individually each turbine has a diameter of 7.6 $\mathrm{cm}$

Left wall

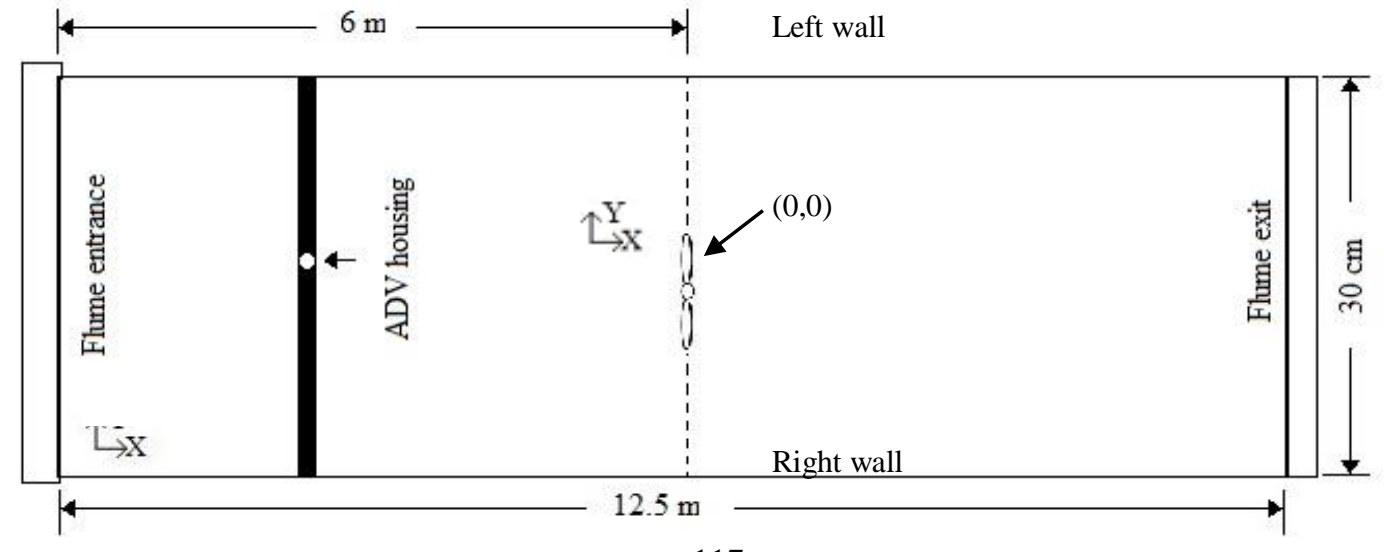


The turbines are placed in staggered position as

Figure 3. The effect of the of the lateral (X) and longitudinal (Y) spacing effect on the flow has been conducted by changing the lateral and

Figure 3. The transverse spacing between the second-row turbines has been varied between $1.5 \mathrm{D}$ $(11.4 \mathrm{~cm})$ and $2 \mathrm{D}(15.2 \mathrm{~cm})$.

Figure 1 Top view of the flume and the position of the first turbine in the array

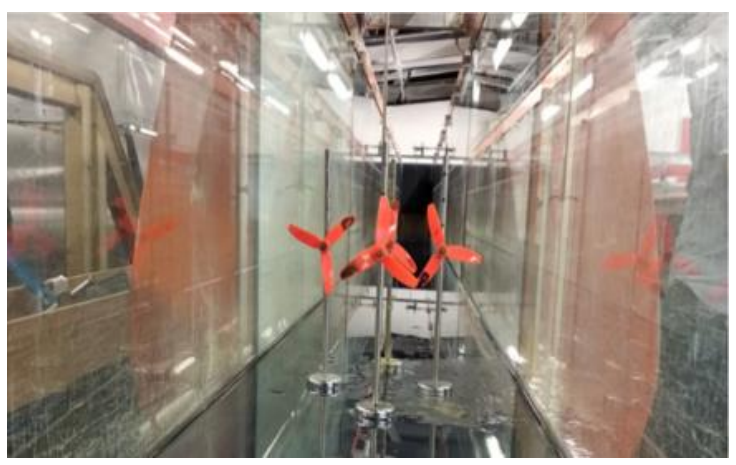

Figure 2 The model turbine used in the experiments

Whereas, the longitudinal spacing between the second and third turbine has been changed between $3 \mathrm{D}(22.8 \mathrm{~cm})$ and $6 \mathrm{D}(45.72 \mathrm{~cm})$. The experiments shown

in

longitudinal distance in the second and third row of

the

array

as shown

in

were denoted by a symbol that showed both the longitudinal (L1) and lateral (T1) spacing of the experiment, these symbols, are used in the discussion of the results to differentiate the experiment. For example, E3,1.5 refers to a 3D longitudinal spacing between the second and the third row in the array, and 1.5D lateral spacing between the centre turbines in the second row

\subsection{Flow conditions:}

Changing the fluid properties is impractical for testing equipment and increasing the velocity will require the blade to operate at high rotational velocity. Thus, Froude number $\left(\mathrm{F}_{\mathrm{r}}\right)$ is the ratio of the inertia to the gravitational force as in [26]

$$
F r=\frac{U_{0}}{\sqrt{g h}}
$$

where $\mathrm{U}$ is the flow velocity, $\mathrm{g}$ is the acceleration due to gravity and $h$ is the water depth. Froude number becomes of high importance when the gravitational effect cannot be ignored. This happens when the free surface is in close proximity to the blade. The Froude number is taken as .22 for this study. This number is adopted from the range specified for different turbines arrangements in [34].

\subsection{Data representation:}

The wake recovery can be indicated by using a parameter known as velocity deficit. The Velocity deficit indicates the rate of recovery when it is plotted downstream of a tidal turbine or an array of tidal turbines

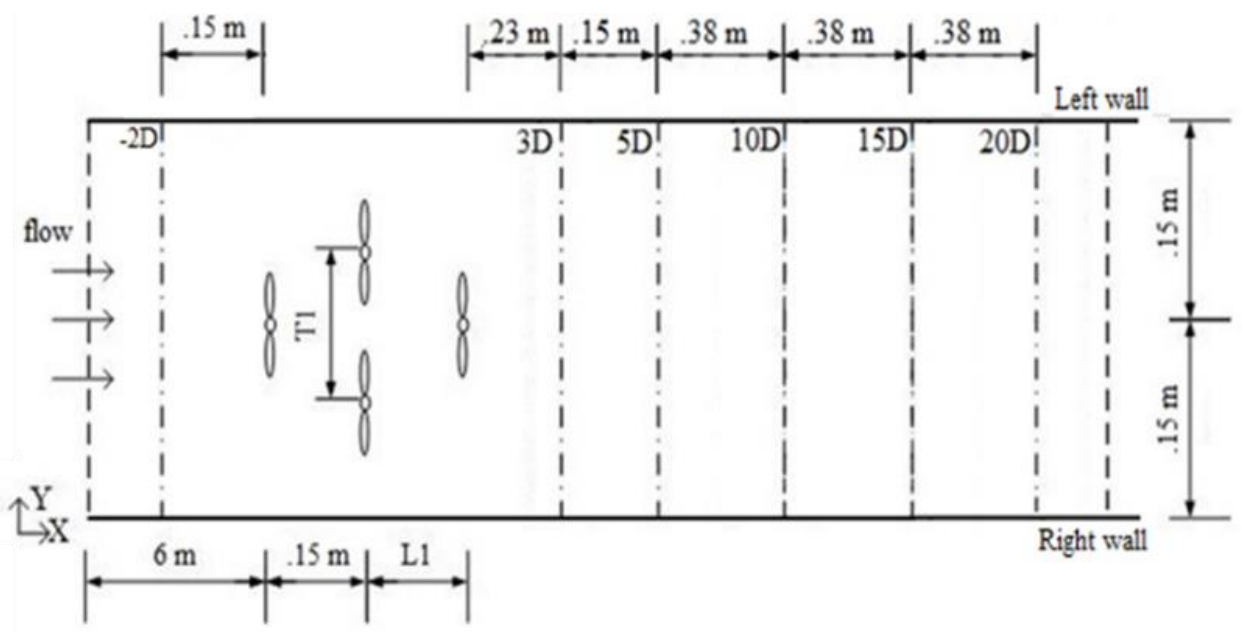


Figure 3 Top view of turbine arrangements and transverse measuring points along the flume

This parameter which can be calculated as,

$U_{\text {defx }}=1-\frac{U_{x}}{\overline{\bar{U}_{0}}}$

where $U_{\text {defx }}$ is the longitudinal velocity deficit, $\bar{U}_{0}$ is the upstream cross-sectional average velocity in the longitudinal direction. Whereas the turbulence intensity is normally calculated as:

$T I=\frac{\sqrt{\frac{1}{3}\left(u^{\prime 2}+{v^{\prime}}^{2}+w^{\prime 2}\right)}}{\bar{U}_{0}}$

Where $\left(u^{\prime}\right),\left(v^{\prime}\right)\left(w^{\prime}\right)$, are the root mean square (RMS) of the turbulent velocity fluctuations in the longitudinal, lateral and vertical directions.
The results are represented in the lateral and vertical profiles

\subsection{Longitudinal profile}

3.1.1 Wake recovery

The highest velocity deficit in Error! Reference source not found., is observed in the near wake region behind the turbine at $3 \mathrm{D}$ from the last turbine, with a slower rate of recovery up to $5 \mathrm{D}$. The cause for the high values of velocity deficit in the near region following the turbine could be attributed to the blockage effect, which decreases with the increase in the downstream distances [35]. The profile of the rate of recovery of velocity in all experiments is comparable to the previous studies in [14],[36].

\section{RESULTS}

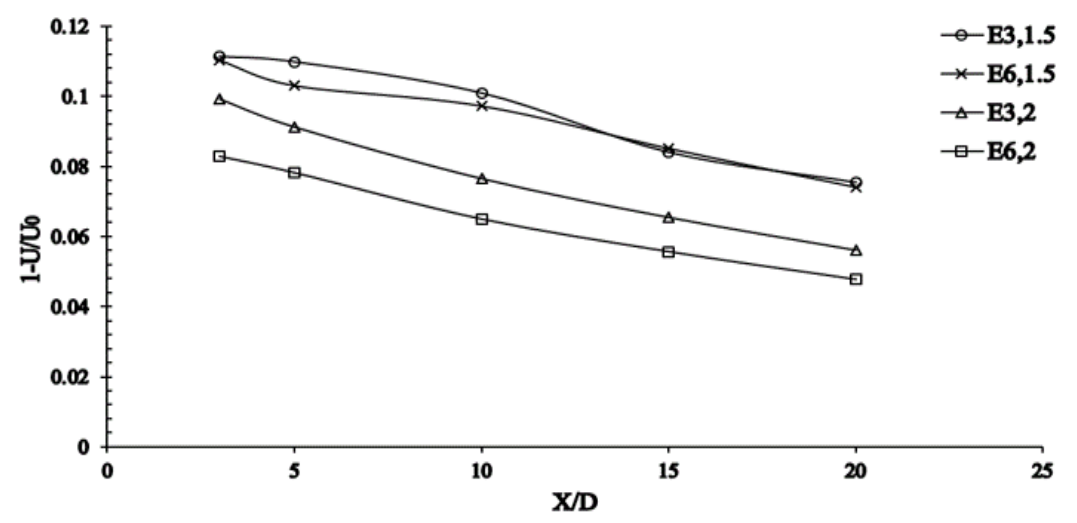

Figure 4: Longitudinal variation of velocity deficit downstream of the array

Increasing the distance in the lateral direction increases the rate of recovery of the velocity i.e. lesser values of velocity deficit, as shown in Error! Reference source not found..

Conversely, this is not clear when the distance between the central turbines and the third row of the array increases in the longitudinal direction.

The small transverse spacing results in the adjacent wakes to form a wide single wake that decreases the rate of wake energisation as noticed in the work of [12]. The profiles of 1.5D spacing in the lateral direction, in experiments $\mathrm{E} 3,1.5$ and E6,1.5, are almost identical and they show a faster rate of recovery after $5 \mathrm{D}$ in the longitudinal direction. On the other hand, the profile of 2D spacing in the lateral direction shown in experiments E3,2 and E6,2, show a better rate of recovery in comparison to the previous spacing lateral spacing.

\subsubsection{Turbulence intensity}

The values of the turbulence intensity are higher in the region behind the turbine, due to the combined effect of the rotor and the structure. The same could be observed in the work of [15]. There is a reduction in the values of the turbulence intensity with the increase of the downstream distances. This reduction is a result of the decline in the turbulent kinetic energy.

The difference in the turbulence intensity values is perceptible up to 5D after which, the values are almost the same, as the turbulence intensity levels approach the upstream level.

All in all, the increase in the transverse spacing lead to higher turbulence and a better wake recovery. Whereas increasing the lateral spacing reduces the turbulences and increase the wake 


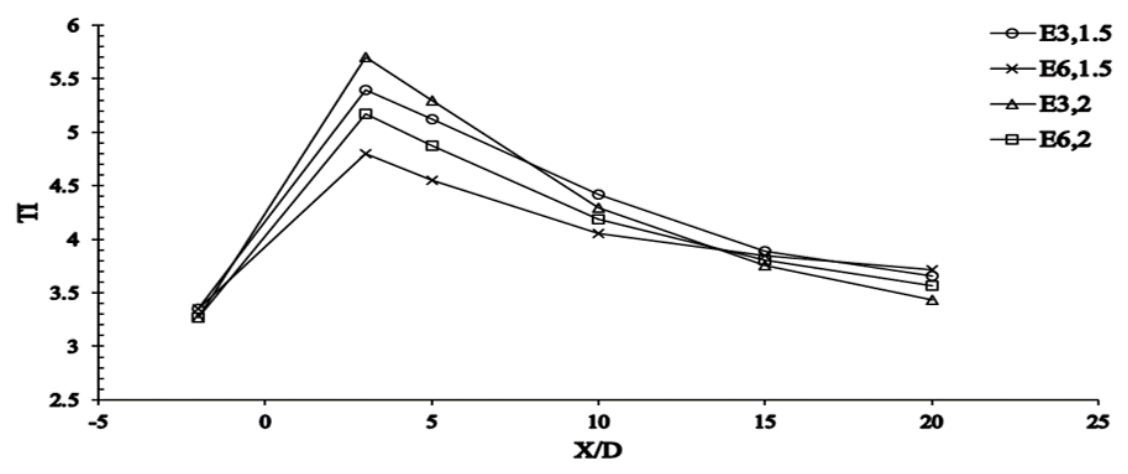

Figure 5: Longitudinal variation of turbulence intensity

\subsection{Lateral profile}

The lateral profiles of the velocity deficit are shown in Figure 5.5. The wake profile is approximately symmetrical in all experiments with the highest velocity deficit occurring at the centreline of the array. As the distance increases in the direction of the flow the rate of velocity recovery increases. The velocity profile at $20 \mathrm{D}$ has a comparable profile to the upstream profile of the turbine shown at 2D in front of the array. However, the velocity has not fully recovered. Moreover, the effect of the second row is clear in the edges of the curves of the $3 \mathrm{D}$ and $5 \mathrm{D}$ of experiments $\mathrm{E} 3,1.5$ and $\mathrm{E} 3,2$ and lesser extent in E6,1.5 and E6,2.The wall effect is much clearer in E3,2 (Error! Reference source not found.-b) and E6,2 (Error! Reference source not found.-d)

(a)

(b)
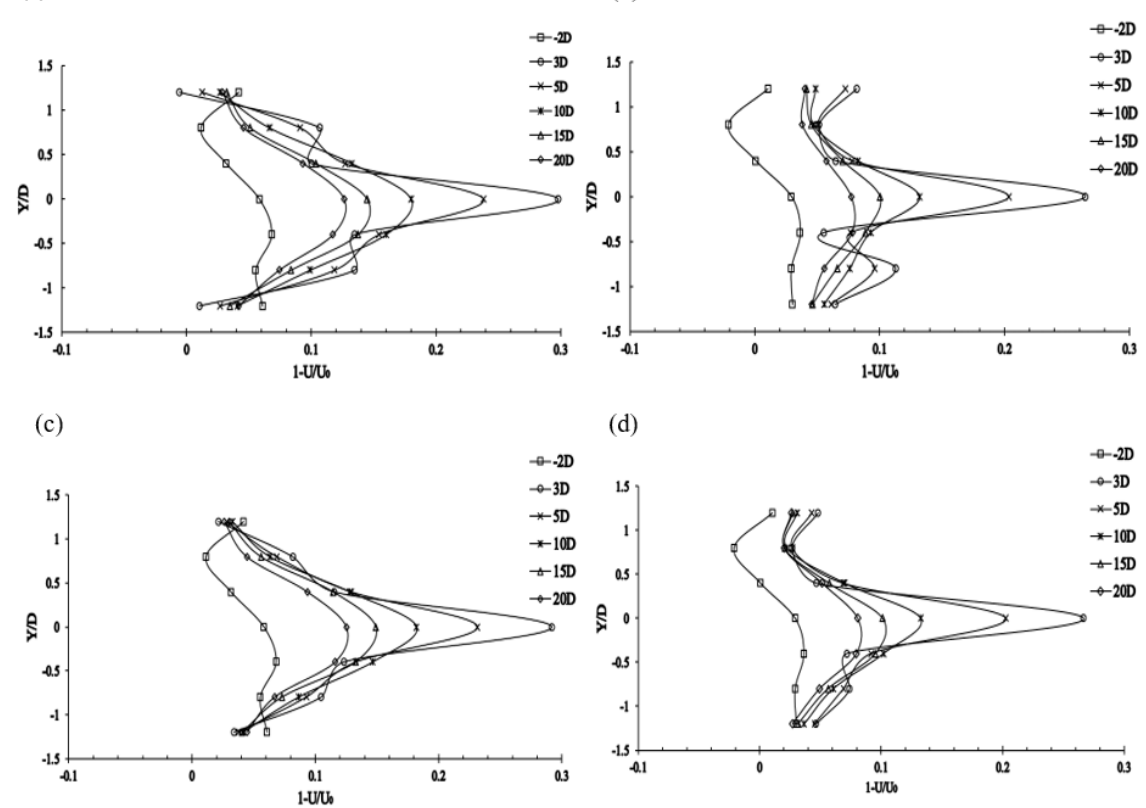

Figure 6 : Lateral variation of velocity deficit in (a) E3,1.5, (b) E3,2, (c) E6,1.5, (d) E6,2

because of the reduced space between the centre turbines and the wall. This visible in the right wall where the flow is decelerating. The figures resemble the profiles of three turbines in [37], with composite blades [38] where a single turbine is put in the rear. It is noticeable that the wakes start to merge at $10 \mathrm{D}$ and completely merge at $15 \mathrm{D}$. To summarize, the increase in the lateral spacing between the centre turbines leads to a faster recovery rate, as shown when comparing E6,2 and $\mathrm{E} 3,2$ with $\mathrm{E} 6,1.5$ and $\mathrm{E} 6,2$.

Moreover, the profiles for the array layouts that have the same lateral profile is almost identical, in 
contrast to the experiments with different longitudinal spacing.

\subsection{Vertical profile}

The vertical profile for the velocity deficit is shown in Figure 5.6. The measurements are taken from 2D upstream to 20D downstream. At 3D there is a large velocity deficit at the centre line. The velocity deficit reduces at this location to approach the

(a)

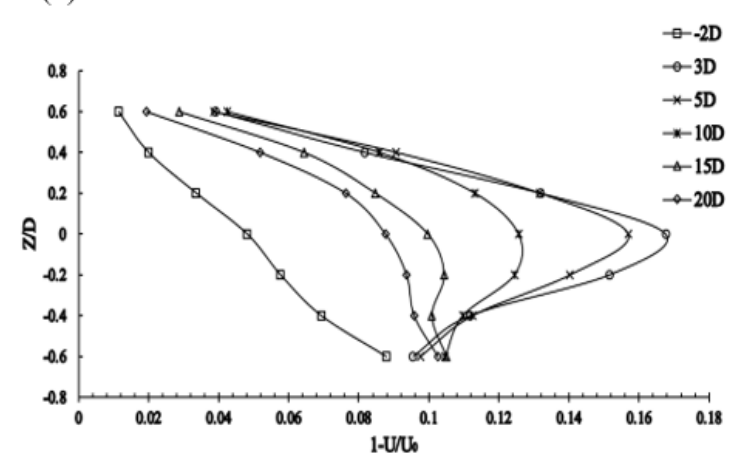

(c)

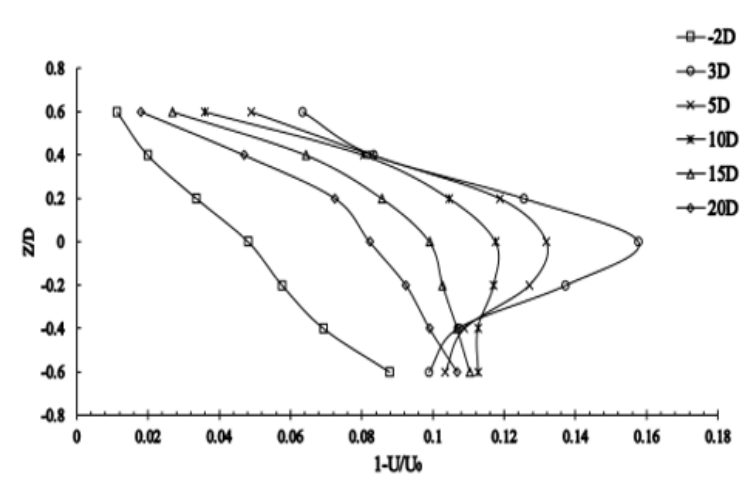

upstream velocity at the tip of the blade above the rotor's centreline. However, the velocity deficit profile is not symmetrical around the rotor centre. Below the centreline, the velocity seems to be slower, and the velocity deficit is higher than above the rotor centre. Nevertheless, this could be because of the lower part of the turbine rotor is close to the

(b)

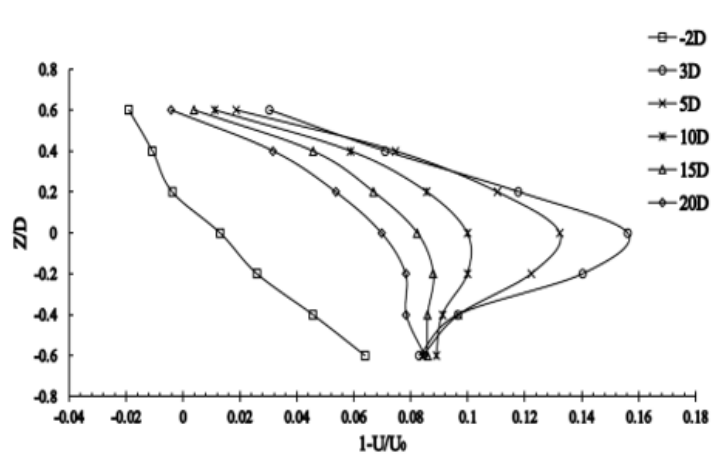

(d)

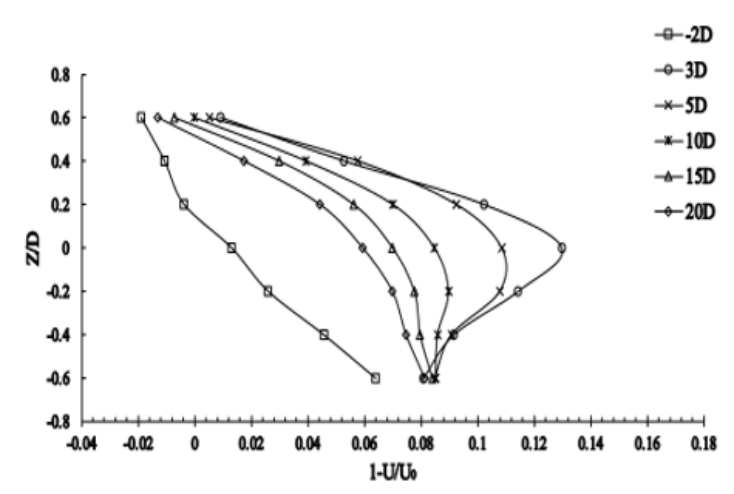

Figure 4: Vertical profile of velocity deficit (a) E3,1.5, (b) E3,2, (c) E6,1.5, (d) E6,2

flume floor. All the experiments seem to have the same profile, were further downstream the velocity deficit measurements seem to decline. The rate of decline is high between $5 \mathrm{D}$ and 10D, and then the rate slows down for the remainder measurements downstream. The lowest velocity deficit occurs at $20 \mathrm{D}$, where the shape velocity deficit profile approaches the upstream velocity profile. The increase in both the lateral and longitudinal spacing had a positive effect on the vertical velocity recovery as in E6,2.

\section{CONCLUSIONS}

The results showed that for all experiments there was a faster rate of recovery from $5 \mathrm{D}$ to $20 \mathrm{D}$ downstream of the array in the longitudinal direction. In these experiments, increasing the lateral distance while maintaining the same longitudinal distance between the turbines in the second row and third row, lead to a better rate of recovery and higher turbulence intensity values at $3 \mathrm{D}$ behind the turbine In the lateral profiles of the velocity deficit, in all experiments, the effect of the second-row turbines seem to disappear after 5D downstream of the array.

Further, the highest velocity deficit occurred at the centre line of the array 3D downstream of the array. Moreover, changing the lateral distance between turbines resulted in a faster rate of recovery in the lateral profile. The same trend happened in the vertical profiles of the velocity deficit when changing the lateral distance between the turbines. The distribution of the turbulence intensities appears to be concentrated in the middle of the cross-sections of 3D and 5D. After 5D, the distribution is pattern less, because of the rapid mixing between the wakes and the surrounding 
flow. The highest values of the turbulence intensity are at cross-section 3D downstream of the array.

However, the array with the smallest lateral and longitudinal distances between the second and third row had the highest values distributed at this crosssection. The drag force coefficient seems to be affected by lateral distances, as the increasing the lateral distance lead to lower values of drag force. All in all, increasing the lateral distance lead to better velocity deficit profiles and lower drag coefficients.

\section{REFERENCES}

[1] A. Bahaj, W. Batten and G. McCann, "Experimental verifications of numerical predictions for the hydrodynamic performance of horizontal axis marine current turbines," Renewable Energy, vol. 32, (15), pp. 2479-2490, 2007.

[2] Z. Zhou et al, "Developments in large marine current turbine technologies-A review," Renewable and Sustainable Energy Reviews, vol. 71, pp. 852858, 2017.

[3] M. Nuernberg and L. Tao, "Experimental study of wake characteristics in tidal turbine arrays," Renewable Energy, vol. 127, pp. 168-181, 2018.

[4] Z. Chen, "Power electronic converter systems for direct drive renewable energy applications," in Electrical Drives for Direct Drive Renewable Energy Systems Anonymous Elsevier, pp. 106-135, 2013.

[5] F. Boyle, A. Reynolds and F. O. Rourke, "Tidal energy update 2009," in Applied Energy, 2010.

[6] M. Elamin, B. Li and K. Tan, "Impact damage of composite sandwich structures in arctic condition", Composite Structures, vol. 192, pp. 422-433, 2018.

[7] M. Khan, M. Elamin, B. Li and K. Tan, "X-ray micro-computed tomography analysis of impact damage morphology in composite sandwich structures due to cold temperature arctic condition", Journal of Composite Materials, vol. 52, no. 25, pp. 3509-3522, 2018.

[8] K. Tan, M. Elamin and B. Li, "Impact Performance and Damage Behavior of Composite Sandwich Structures in Arctic Condition", in American Society for Composites-Thirty-second Technical Conference, 2017.
[9] M. Elamin, B. Li and K. Tan, "Impact Performance of Stitched and Unstitched Composites in Extreme Low Temperature Arctic Conditions", Journal of Dynamic Behavior of Materials, vol. 4, no. 3, pp. 317-327, 2018.

[10] M. Elamin and J. Varga. "Plate impact method for shock physics testing," Material Sci \& Eng, vol. 4, no. 1, pp. 31-35, 2020.

[11] S. P. Neill and M. R. Hashemi, Fundamentals of Ocean Renewable Energy: Generating Electricity from the Sea. Academic Press, 2018.

[12] N. K. Stelzenmuller, Marine Hydrokinetic Turbine Array Performance and Wake Characteristics, 2013.

[13] R. Vennell, "Tuning tidal turbines in-concert to maximise farm efficiency," J. Fluid Mech., vol. 671, pp. 587-604, 2011.

[14] Y. Chen et al, "Experimental study of wake structure behind a horizontal axis tidal stream turbine," Appl. Energy, vol. 196, pp. 82-96, 2017.

[15] T. Stallard et al, "Interactions between tidal turbine wakes: experimental study of a group of three-bladed rotors," Philosophical Transactions of the Royal Society A: Mathematical, Physical and Engineering Sciences, vol. 371, (1985), pp. 20120159, 2013.

[16] M. J. Churchfield, Y. Li and P. J. Moriarty, "A large-eddy simulation study of wake propagation and power production in an array of tidal-current turbines," Philosophical Transactions of the Royal Society A: Mathematical, Physical and Engineering Sciences, vol. 371, (1985), pp. 20120421, 2013.

[17] X. Sun, J. Chick and I. Bryden, "Laboratoryscale simulation of energy extraction from tidal currents," Renewable Energy, vol. 33, (6), pp. 1267-1274, 2008.

[18] A. MacLeod et al, "Wake effects in tidal current turbine farms," in International Conference on Marine Renewable Energy-Conference Proceedings, pp. 49-53, 2002.

[19] T. Stallard and S. Gant, "Modelling a tidal turbine in unsteady flow," in The Eighteenth International Offshore and Polar Engineering Conference, 2008, .

[20] M. H. Ahmadi, "Influence of upstream turbulence on the wake characteristics of a tidal 
stream turbine," Renewable Energy, vol. 132, pp. 989-997, 2019.

[21] M. Harrison, W. Batten and A. Bahaj, "A blade element actuator disc approach applied to tidal stream turbines," in Oceans 2010 Mts/Ieee Seattle, pp. 1-8, 2010.

[22] M. G. Gebreslassie, G. R. Tabor and M. R. Belmont, "Investigation of the performance of a staggered configuration of tidal turbines using CFD," Renewable Energy, vol. 80, pp. 690-698, 2015.

[23] G. Bai et al, "Numerical investigations of the effects of different arrays on power extractions of horizontal axis tidal current turbines," Renewable Energy, vol. 53, pp. 180-186, 2013.

[24] A. Bahaj et al, "Power and thrust measurements of marine current turbines under various hydrodynamic flow conditions in a cavitation tunnel and a towing tank," Renewable Energy, vol. 32, (3), pp. 407-426, 2007.

[25] T. O'Doherty et al, "Experimental and computational analysis of a model horizontal axis tidal turbine," in 8th European Wave and Tidal Energy Conference (EWTEC), Uppsala, Sweden, 2009 . .

[26] L. Myers and A. Bahaj, "Experimental analysis of the flow field around horizontal axis tidal turbines by use of scale mesh disk rotor simulators," Ocean Eng., vol. 37, (2-3), pp. 218$227,2010$.

[27] F. Maganga et al, "Experimental characterisation of flow effects on marine current turbine behaviour and on its wake properties," IET Renewable Power Generation, vol. 4, (6), pp. 498509, 2010.

[28] S. Tedds, I. Owen and R. Poole, "Near-wake characteristics of a model horizontal axis tidal stream turbine," Renewable Energy, vol. 63, pp. 222-235, 2014.

[29] Y. Chen et al, "Experimental study of wake structure behind a horizontal axis tidal stream turbine," Appl. Energy, vol. 196, pp. 82-96, 2017.

[30] P. Mycek et al, "Experimental study of the turbulence intensity effects on marine current turbines behaviour. Part I: One single turbine," Renewable Energy, vol. 66, pp. 729-746, 2014.
[31] P. Mycek et al, "Experimental study of the turbulence intensity effects on marine current turbines behaviour. Part II: Two interacting turbines," Renewable Energy, vol. 68, pp. 876-892, 2014.

[32] S. Chawdhary et al, "Wake characteristics of a TriFrame of axial-flow hydrokinetic turbines," Renewable Energy, vol. 109, pp. 332-345, 2017.

[33] L. Myers and A. Bahaj, "An experimental investigation simulating flow effects in first generation marine current energy converter arrays," Renewable Energy, vol. 37, (1), pp. 28-36, 2012.

[34] NORTEK, "CollectV software manual," USA, 2000.

[35] S. Tedds, Scale Model Testing of Tidal Stream Turbines: Wake Characterisation in Realistic Flow Conditions, 2014.

[36] J. Thomson et al, "Quantifying turbulence for tidal power applications," in Oceans $2010 \mathrm{Mts} / \mathrm{Ieee}$ Seattle, 2010, pp. 1-8.

[37] M. Nuernberg and L. Tao, "Experimental study of wake characteristics in tidal turbine arrays," Renewable Energy, vol. 127, pp. 168-181, 2018.

[38] M. Elamin, B. Li, and K.T. Tan, "EFFECT OF LOW TEMPERATURE ON IMPACT BEHAVIOR OF COMPOSITE SANDWICH STRUCTURES." 